\title{
A survey on the resources and practices in pediatric critical care of resource-rich and resource-limited countries
}

\author{
Sandeep Tripathi ${ }^{4 *}$, Harsheen Kaur ${ }^{1}$, Rahul Kashyap ${ }^{2}$, Yue Dong $^{2}$, Ognjen Gajic ${ }^{2}$ and Srinivas Murthy ${ }^{3}$
}

\begin{abstract}
Background: Contemporary critical care research necessitates involvement of multiple centers, preferably from many countries. Adult and pediatric research networks have produced outstanding data; however, their involvement is restricted to a small percentage of the industrialized nations. Implementation of their findings in low- and middle-income countries (LMICS) is fraught with challenges.

Methods: We conducted an online international survey to assess and compare disease burden and resources to participate in multicenter research studies through a listserv of the World Federation of Pediatric Intensive and Critical Care Societies. Respondents were grouped into high-income countries and LMICs on the basis of World Bank classification.

Results: Survey was completed by 73 centers in 34 countries (34 from high-income countries and 39 from LMICs). Compared with high-income countries, the pediatric intensive care units in LMICs were characterized by a lower number of critical care specialists, more difficult access to hemodialysis, and a lower number of elective postoperative patients, but a similar overall disease burden. Training and resources for research were comparable in the two cohorts.

Conclusions: Although differences exist in access to both trained providers and equipment, the survey results were more striking in their similarity. It is essential that centers from LMICs be included in multinational studies, to generate results applicable to all children worldwide.
\end{abstract}

Keywords: Pediatric critical care, International survey, Low- and middle-income countries

\section{Background}

The delivery of pediatric critical care $(\mathrm{CC})$ in resourcerich regions has progressed dramatically over recent decades, with markedly improved outcomes for critically ill children around the planet. Many of the advances in the care of critically ill children have been through high-cost interventions, with enhanced accessibility to resources, trained staff, and new technology. However, the vast majority of the world's child population and pediatric disease burden are in regions where resource limitations are more prevalent [1]. There are scant data about the nature of global pediatric $\mathrm{CC}$, especially in resourcelimited regions. Given the infrastructure required to maintain a $\mathrm{CC}$ unit, ensuring personnel and equipment

\footnotetext{
* Correspondence: sandeeptripathi2000@yahoo.com

${ }^{4}$ Department of pediatrics, University of Illinois at Chicago, Peoria, IL, USA Full list of author information is available at the end of the article
}

availability is a tremendous challenge in resource-limited economies.

A few reports have addressed the adult $\mathrm{CC}$ services and utilization in developing countries [2]. However, little is known about the international differences in pediatric CC services. Although individual countries have previously published data on the resources [3, 4], no large international study has compared resources among the different parts of the world. In addition, much of the CC evidence base has come from resourcerich countries [5]; research from low- and middle-income countries (LMICs) represents only a small fraction of $\mathrm{CC}$ research performed around the planet [6-8].

Incorporation of evidence from different regions of the world with different health systems is fraught with difficulty. Evidence generated within a sole country or across countries is often implemented in the care of critically ill 
patients worldwide without further local testing [9]. Research done in resource-rich regions, when reproduced in other countries, has led to contradictory results [10]. Therefore, it is essential that high-quality research be carried out in regions where disease burden is high, to apply locally generated evidence.

The present survey was conducted to identify the pediatric $\mathrm{CC}$ resources and to research the infrastructure available in resource-limited countries as part of recruitment efforts for a multicentric research study to evaluate the automated rounding and clinical decision support tool, Checklist for Early Recognition and Treatment of Acute Illness in Pediatrics (CERTAINp) [11]. We describe herein the secondary data analysis of survey results.

\section{Methods}

We adapted a survey for pediatric CC providers from a previously published and validated Web-based survey [12]. The resulting cross-sectional survey consisted of 138 questions containing numeric, binomial, and categorical questions, as well as descriptive questions. The survey was administered to email addresses obtained from the email list of the World Federation of Pediatric Intensive and Critical Care Societies. This list contained more than 6000 persons (physicians, nurses, midlevel providers, and other health care professionals) active in pediatric intensive care from around the world, with representation from every inhabited continent. The survey was conducted in November and December 2014.

Respondents were asked to classify the various diseases and conditions in the intensive care unit (ICU) on a spectrum of very common to very uncommon, including rank-order lists of causes of death in their hospital. Access to $\mathrm{CC}$ resources, such as equipment, skilled staff, and medications, was categorized on a scale from very difficult to very easy. All answers were based on the perceptions of the respondent individually. Hospital location was classified as resource-rich or resource-limited on the basis of World Bank classification [13]. High-income (gross national income per capita, >US\$12,746) countries (HICs) were classified as resource-rich. By comparison, the other areas were classified as upper-middle-income countries (UMICs) (gross national income, US\$4126US $\$ 12,745$ in 2013), LMICs (gross national income, US\$1046-US\$4125), and low-income countries (LICs) (gross national income, $\leq \mathrm{US} \$ 1045$ ) as resource-limited.

\section{Statistical analysis}

Raw data from the survey were exported to an electronic file for analysis (JMP; SAS Institute Inc.). Numeric values, such as the number of pediatric beds in a hospital or the number of intensivists, were analyzed as mean (SD) and compared between the two groups with $t$ test. Binomial variables such as presence or absence of a research coordinator or of formal CC training were analyzed with $\chi^{2}$ test as proportions. Categorical variables with more than two choices were dichotomized into two groups for analysis with $\chi^{2}$ test. Because of the large number of questions, certain related fields were grouped together for analysis. The study was approved by the Mayo Clinic Institutional Review Board as part of the multicentric CERTAINp study. Need for informed consent was waived. There were no patients enrolled in the survey and no patient-specific data was collected.

\section{Results}

The survey was emailed twice-on November 5 and November 27, 2014-to 6172 and 6141 respondents. The emails were read by 1838 and 1742 recipients, with 326 and 245 opening the survey. A total of 73 hospitals (12.9\% response rate) completed the survey, representing 34 countries: 15 HICs, 14 UMICs, 3 LMICs, and 2 LICs (15 resource-rich and 19 resource-limited). All responses were from separate institutions. Pediatric age range in the earlier study and suggested for this survey was birth to 18 years.

\section{ICU and research capacity}

The mean (SD) total numbers of pediatric beds were comparable in the two groups $(P=.79)$ (Table 1). The numbers of self-defined CC beds $(P=.007)$ and ICU admissions $(P=.03)$ were significantly greater in HICs. Among centers in resource-limited regions, $51.2 \%$ had a research coordinator and $66.6 \%$ had a dedicated quality

Table 1 Comparison of the resources between two groups of countries in accordance with survey responses

\begin{tabular}{llll}
\hline Resource $^{\text {a }}$ & $\begin{array}{l}\text { Developed } \\
\text { country }(n=34)\end{array}$ & $\begin{array}{l}\text { Developing } \\
\text { country }(n=39)\end{array}$ & $P$ value \\
\hline Pediatric beds & $169(88.4)$ & $158(211)$ & .79 \\
Critical care beds & $17.5(11.5)$ & $11.2(6.8)$ & .007 \\
Admissions/year in PICU & $870.4(548)$ & $600.1(504)$ & .03 \\
PICU physicians & $10.0(5.8)$ & $5.5(4.3)$ & $<.001$ \\
EMR & $85.29(29)$ & $48.72(19)$ & .001 \\
Dedicated research & $47.06(16)$ & $51.28(20)$ & .72 \\
coordinator & & & .36 \\
Dedicated QI program & $76.47(26)$ & $66.67(26)$ & .36 \\
Reliable Internet access & $97.06(33)$ & $87.18(34)$ & .13 \\
Critical care specialization & $94.12(32)$ & $94.87(37)$ & .89 \\
Prior participation in & $85.2(29)$ & $58.9(23)$ & .13 \\
multicentric trials & & & .26 \\
ACLS/PALS & $91.18(31)$ & $82.05(32)$ & .75 \\
\hline PFCCS & $32.35(11)$ & $35.90(14)$ & \\
\hline
\end{tabular}

ACLS advanced cardiac life support, EMR electronic medical record, $P A L S$ pediatric advanced life support, PFCCS pediatric fundamental critical care support, $P I C U$ pediatric intensive care unit, $Q$ l quality improvement

${ }^{a}$ Numerical values are presented as number (SD); categorical values are presented as percentage and number of "Yes" responses 
improvement program. No difference was found in the availability of reliable Internet access in the ICU between the two regions. Resource-limited regions also had limited implementation of electronic medical records $(P=.001)$. A higher proportion of centers from HICs had participated in prior multicentric trials; however, this difference was not statistically significant $(P=.13)$.

\section{ICU staffing}

Almost twice the number of intensivists (i.e., physicians primarily practicing in the pediatric intensive care unit) work in each unit in the resource-rich countries (mean [SD], 10.0 [5.8] vs 5.5 [4.3]). However, there was comparable staff (doctor or nurse) with $\mathrm{CC}$ training (CC specialization, advanced cardiac life support, pediatric advanced life support, or pediatric fundamental CC support; $P=.89$ ) (Table 1 ). Access to the different services of CC delivery was not reported as difficult or very difficult in industrialized countries, and ICU-trained nurses and occupational and physical therapists were significantly less readily available in resource-limited regions (23.08 and $17.95 \%$ reporting difficult or very difficult, respectively) (Table 2). Among all the support services, respiratory therapists were the most difficult to access, even in HICs and LMICs (reported difficult or very difficult, 20.59 vs $38.46 \% ; P=.09$ ). An anesthesiologist was the only medical provider with a significant difference in difficulty to access between the two groups: No center from resource-rich countries reported difficulty, whereas $12.82 \%$ from resource-limited regions reported either difficult or very difficult access.

\section{ICU resources}

Although all survey-included ICU equipment was harder to access in resource-limited countries, only central intravenous catheter $(P=.03)$, ICU monitoring equipment

Table 2 Difficulty in the availability of trained staff and finances

\begin{tabular}{llll}
\hline Trained staff $^{\mathrm{a}}$ & $\begin{array}{l}\text { Developed country, } \\
\%(\mathrm{no} .)(n=34)\end{array}$ & $\begin{array}{l}\text { Developing country, } \\
\%(\mathrm{no} .)(n=39)\end{array}$ & $P$ value \\
\hline Anesthesiologist & $0(0)$ & $12.82(5)$ & .03 \\
Surgeon & $0(0)$ & $2.56(1)$ & .35 \\
ICU specialist & $0(0)$ & $7.69(3)$ & .10 \\
Nurse & $0(0)$ & $2.56(1)$ & .35 \\
ICU-trained nurse & $0(0)$ & $23.08(9)$ & .002 \\
Pharmacist & $2.94(1)$ & $12.82(5)$ & .13 \\
OT/PT & $2.94(1)$ & $17.95(7)$ & .04 \\
RT & $20.59(7)$ & $38.46(15)$ & .09 \\
Finances & $2.94(1)$ & $35.90(14)$ & $<.001$ \\
\hline
\end{tabular}

ICU intensive care unit, OT occupational therapist, PT physical therapist, $R T$ respiratory therapist

${ }^{a}$ Values indicate the percentage of respondents in the group who selected "difficult" or "very difficult" for the survey item
$(P=.01)$, radiology services $(P=.009)$, and dialysis equipment $(P<.001)$ were statistically significant (Table 3$)$. No difference was found in access to hospital beds, ICU beds, operating rooms, mechanical ventilators, or cardiac defibrillators. Only three centers from the LMIC group reported difficulty in access to crystalloid fluids (normal saline or lactated Ringer solution). Accessing antimicrobials was difficult in $25.6 \%$ of resource-limited regions, compared with $14.7 \%$ of resource-rich regions. Cardiovascular medications were difficult to access in $2.5 \%$ of resourcelimited regions vs $0 \%$ in resource-rich regions. Opioids, benzodiazepines, and ketamine were significantly more difficult to access in resource-limited regions (23.08 vs $2.94 \%$, $P=.01)$. For $23.08 \%$ of respondents in resource-limited regions, blood products were difficult to obtain. Finances continue to be a challenge in these regions as well, with $35.9 \%$ of the centers struggling for adequate financial resources compared with $2.9 \%$ in HIC reporting difficulty with finances (data not shown).

\section{Admitting diagnosis and causes of death}

Malnutrition continues to be prevalent in resourcelimited countries, as suggested by $35.9 \%$ of the countries reporting very frequent nutritional diseases in the ICU (Table 4). Tuberculosis (28.1\%), malaria (10.2\%), human immunodeficiency virus $(10.2 \%)$, and rheumatic heart disease $(7.7 \%)$ were specific to resource-limited regions. The ICUs of resource-rich regions reported a

Table 3 Difficulty in the availability of ICU equipment and medications

\begin{tabular}{llll}
\hline Equipment and medication $^{\mathrm{a}}$ & $\begin{array}{l}\text { Developed } \\
\text { country }(n=34)\end{array}$ & $\begin{array}{l}\text { Developing } \\
\text { country }(n=39)\end{array}$ & $P$ value \\
\hline Hospital beds & $0(0)$ & $10.26(4)$ & .05 \\
ICU beds & $2.94(1)$ & $12.82(5)$ & .13 \\
Operating room & $0(0)$ & $5.13(2)$ & .10 \\
Central IV catheter & $0(0)$ & $12.82(5)$ & .03 \\
ICU monitoring devices & $2.94(1)$ & $23.08(9)$ & .01 \\
Mechanical ventilators & $0(0)$ & $7.69(3)$ & .10 \\
Cardiac defibrillators & $0(0)$ & $5.13(2)$ & .18 \\
Radiology equipment & $0(0)$ & $17.95(7)$ & .009 \\
Dialysis equipment & $2.94(1)$ & $38.46(15)$ & $<.001$ \\
Blood bank & $0(0)$ & $5.13(2)$ & .18 \\
Antimicrobials & $14.71(5)$ & $25.64(10)$ & .25 \\
Pain/sedation medications & $2.94(1)$ & $23.08(9)$ & .01 \\
Cardiovascular drugs & $0(0)$ & $2.56(1)$ & .35 \\
Crystalloid fluids & $0(0)$ & $7.69(3)$ & .09 \\
Blood products/albumin & $0(0)$ & $23.08(9)$ & .002 \\
\hline
\end{tabular}

ICU intensive care unit, $I V$ intravenous

aPercentage of respondents in the group who selected "difficult" or "very difficult" for the survey item

${ }^{\mathrm{b}}$ These medications include opioids, benzodiazepine, and ketamine 
Table 4 The most common disease states cited by survey respondents

\begin{tabular}{llll}
\hline Disease state $^{\text {a }}$ & $\begin{array}{l}\text { Developed } \\
\text { country }(n=34)\end{array}$ & $\begin{array}{l}\text { Developing } \\
\text { country }(n=39)\end{array}$ & $P$ value \\
\hline Toxins & $11.76(4)$ & $12.82(5)$ & .89 \\
Newborn illness & $41.18(14)$ & $30.77(12)$ & .35 \\
Tuberculosis & $0(0)$ & $28.21(11)$ & $<.001$ \\
Malaria & $0(0)$ & $10.26(4)$ & .05 \\
HIV infection & $0(0)$ & $10.26(4)$ & .05 \\
Rheumatic heart disease & $0(0)$ & $7.69(3)$ & .10 \\
Congenital heart disease & $44.12(15)$ & $43.59(17)$ & .96 \\
Nutritional disease & $.05(0)$ & $35.90(14)$ & $<.001$ \\
Cancer & $35.29(12)$ & $17.95(7)$ & .09 \\
Respiratory disease & $88.24(30)$ & $87.18(34)$ & .89 \\
Elective operation & $64.71(22)$ & $33.33(13)$ & .007 \\
Emergency operation & $47.06(16)$ & $30.77(12)$ & .15 \\
Renal failure & $26.47(9)$ & $17.95(7)$ & .38 \\
Diabetes mellitus & $23.53(8)$ & $7.69(3)$ & .06 \\
Status epilepticus & $23.53(8)$ & $12.82(5)$ & .23 \\
Sepsis & $26.47(9)$ & $20.51(8)$ & .55 \\
Meningitis & $11.76(4)$ & $23.08(9)$ & .21 \\
\hline
\end{tabular}

HIV human immunodeficiency virus

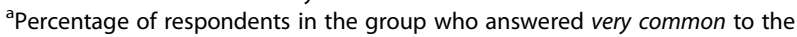
particular disease or syndrome

much higher proportion of patients receiving an elective operation. Respiratory disease and congenital heart disease were the two diseases most frequently cited as very common in both resource-rich and resource-limited regions, representing 88.24 and $87.18 \%$ for respiratory disease and 44.12 and $43.59 \%$ for congenital heart disease, respectively. The top 3 causes of death reported by respondents were similar across income groups: infection, multiorgan dysfunction, and cardiac reasons. Infectious causes of death comprised a greater percentage in the resource-limited countries than resource-rich countries (51.28 vs $23.53 \%, P=.25$ ) (data not shown).

\section{Discussion}

This study reports both large variation and remarkable similarities in CC services across the 34 countries. Pediatric CC providers all over the world continue to struggle with infectious diseases and congenital heart disease. From a research standpoint, many of the survey participants in resource-limited regions appear to have the capacity to participate in clinical research. The lack of resources and higher incidence of infectious diseaserelated deaths in developing countries are expected based on higher birth rate and financial constraints. Our finding of deficits in certain areas, such as staffing and medication access, in resource-limited regions should help guide policy in $\mathrm{CC}$ delivery in these regions.
In the past, several attempts were made to identify overall similarities and differences in CC services; however, most of the studies were limited to a comparison of centers in developed countries [14] or individual countries [15]. International comparisons of pediatric CC delivery have been hampered by the lack of internationally validated outcome prediction scores, given that the primary scores have been validated in resource-rich regions only [16]. Their application to ICU services in varied settings with varied diseases and clinical contexts leads to challenges in interpretation.

A previously published international survey on adult ICU resources in developing countries showed that in a convenience sample of 13 ICUs from resource-limited regions, similar ICU capacities were found (median, 9 beds occupied and 40 patients treated per month; mean [SD] beds, 11.2 [6.8]; mean [SD] admissions per year, 600 [504]), although no resource-rich region comparison occurred [17]. They also observed that standardized processes of care (e.g., checklists) are frequently lacking in these countries. We did not compare specific outcome data or severity of illness among regions, although prior studies have shown higher mortality rates and younger age groups in adult $\mathrm{CC}$ settings in resource-limited regions [7]. As pediatric CC grows increasingly, the capacity in many regions of the world-such as Asia and Africa-to incorporate these data will allow for better evidence guidance.

Given the variability in insurance schemes and health financing in resource-limited regions, CC may be out of reach for most patients who currently are in resourcelimited regions [18]. However, cost-effective CC, where more-than-usual resources are devoted to rescuing severely ill patients, has a fundamental place in any health system and should be a fundamental goal of the field of pediatric CC [19]. This is especially true in pediatrics, where the potential for recovery after acute illness is high and the disease burden is substantial. Rather than a lack of sophisticated ICU technology, a lack of understanding of the CC principles continues to be a major obstacle to good-quality care. Participation in international research and quality improvement efforts can help enhance care delivery. Efforts to create a global registry of worldwide adult ICUs are in process [20], and similar attempts are urgently needed for critically ill children.

The study is a secondary data analysis of an investigation with different objectives, thereby introducing important limitations. Centers with capabilities and interest likely were self-selected and thus may not be representative of the average facilities of the region. However, the fact that 39 centers from resource-limited centers have comparable resources and interest in multicentric clinical trials is encouraging and may help in fostering a clinical trial group composed of the interested centers. 
The information also is self-reported on the basis of perceptions, rather than the clinical records, so an element of bias may be present.

\section{Conclusions}

Absolute CC services differ widely around the planet. Although institutions in HICs clearly have more resources, LMICs have a greater disease burden. This study highlights the differences while showing the similarity and availability of basic infrastructure for services and research. Even after accounting for the limitations, this effort represents an important step to understand the resources available to sick children and for future research initiatives. A critical need exists for ongoing study of health services research in global pediatric CC and for optimization of research settings around the world to better care for the large proportion of the population who are currently affected but do not have access to services.

\section{Key messages}

- First global survey on the resources and manpower in pediatric intensive care.

- Significant disease burden of common pediatric intensive care unit (PICU) conditions in low- and middle-income countries (LMICs).

- Availability of resources and manpower for clinical research exists in developing countries and is comparable to high-income countries at least in some centers.

- To generate data applicable to all the children worldwide, centers from LMICs need to be included in multicentric trials.

\section{Abbreviations}

CC: critical care; CERTAINp: Checklist for Early Recognition and Treatment of Acute IIIness in Pediatrics; HIC: high-income country; ICU: intensive care unit; LIC: low-income country; LMIC: low- and middle-income country;

UMIC: upper-middle-income country.

\section{Competing interests}

The authors declare that they have no competing interests.

\section{Authors' contributions}

ST contributed to the design, analysis, and drafting of the manuscript. HK contributed to the data collection, analysis, and literature search. RK contributed to the design and review of the manuscript. YD contributed to the design and analysis. OG contributed to the concept, design, and review of the manuscript. SM contributed to the data collection, analysis, and drafting of the manuscript. All authors have read and approved this final version of the manuscript.

\section{Disclosure}

This manuscript is not under consideration for publication in any other journal and has not been published before.

\section{Author details}

'Division of Pediatric Critical Care Medicine, Mayo Clinic, Rochester, MN, USA. ${ }^{2}$ Division of Pulmonary and Critical Care Medicine, Mayo Clinic, Rochester, MN, USA. ${ }^{3}$ University of British Columbia, Vancouver, British Columbia, Canada. ${ }^{4}$ Department of pediatrics, University of Illinois at Chicago, Peoria, IL, USA.
Received: 9 June 2015 Accepted: 2 October 2015

Published online: 09 October 2015

\section{References}

1. GBD 2013 Mortality and Causes of Death Collaborators. Global, regional, and national age-sex specific all-cause and cause-specific mortality for 240 causes of death, 1990-2013: a systematic analysis for the Global Burden of Disease Study 2013. Lancet. 2015;385(9963):117-71.

2. Du B, Xi X, Chen D, Peng J, China Critical Care Clinical Trial Group (CCCCTG). Clinical review: critical care medicine in mainland China. Crit Care. 2010;14(1):206. Epub 2010 Feb 25.

3. Kambarami R, Chidede O, Chirisa M. Neonatal intensive care in a developing country: outcome and factors associated with mortality. Cent Afr J Med. 2000;46(8):205-7.

4. Haque A, Ladak LA, Hamid MH, Mirza S, Siddiqui NR, Bhutta ZA. A national survey of pediatric intensive care units in Pakistan. J Crit Care Med. 2014; 1-4. http://dx.doi.org/10.1155/2014/842050. Accessed Oct 6th, 2015.

5. Wunsch H, Harrison D, Collange O, de Keizer N, Fowler R, Hoste E, et al. World resources in critical care study: a survey of critical care research and resources in eight countries. Crit Care. 2007;11 suppl 2:506.

6. Boldt J, Maleck W, Koetter KP. Which countries publish in important anesthesia and critical care journals? Anesth Analg. 1999;88(5):1175-80

7. Adhikari NK, Fowler RA, Bhagwanjee S, Rubenfeld GD. Critical care and the global burden of critical illness in adults. Lancet. 2010;376(9749):1339-46.

8. Murthy S, Leligdowicz A, Adhikari NK. Intensive care unit capacity in low-income countries: a systematic review. PLoS One. 2015;10(1):e0116949.

9. Murthy $\mathrm{S}$, Wunsch $\mathrm{H}$. Clinical review: international comparisons in critical care-lessons learned. Crit Care. 2012;16(2):218.

10. Maitland K, Kiguli S, Opoka RO, Engoru C, Olupot-Olupot P, Akech SO, et al. Mortality after fluid bolus in African children with severe infection. N Engl J Med. 2011;364(26):2483-95.

11. Implementation of a web based real time clinical decision support tool (CERTAINp). www.clinicaltrials.gov (NCT02398981). Accessed Aug 20, 2015.

12. Westcott M, Martiniuk AL, Fowler RA, Adhikari NK, Dalipanda T. Critical care resources in the Solomon Islands: a cross-sectional survey. BMC Int Health Hum Rights. 2012;12:1.

13. The World Bank. Country and Lending Groups [Internet]. c2015. Available from: http://data.worldbank.org/about/country-and-lending-groups. [Assessed 2015 May 18].

14. Wunsch $H$, Angus DC, Harrison DA, Collange $\mathrm{O}$, Fowler $\mathrm{R}$, Hoste EA, et al. Variation in critical care services across North America and Western Europe. Crit Care Med. 2008;36(10):2787-93. e1-9.

15. Odetola FO, Clark SJ, Freed GL, Bratton SL, Davis MM. A national survey of pediatric critical care resources in the United States. Pediatrics. 2005;115(4):e382-6.

16. Wunsch $\mathrm{H}$, Angus DC. International comparisons of intensive care: understanding the differences. In: Vincent $\mathrm{JL}$, editor. Intensive care medicine: annual update 2006. Springer: New York; 2006. p. 786-93.

17. Vukoja M, Riviello E, Gavrilovic S, Adhikari NK, Kashyap R, Bhagwanjee S, et al. A survey on critical care resources and practices in low- and middle-income countries. Glob Heart. 2014;9(3):337-42.e1-5.

18. Gomersall CD. Critical care in the developing world: a challenge for us all. Crit Care. 2010;14(2):131. Epub 2010 Mar 11.

19. Murthy S, Sayeed SA, Adhikari NKJ. Critical care in low resource settings. In: Scales DC, Rubenfeld GD, editors. The organization of critical care: an evidence-based approach to improving quality. Respiratory medicine, vol. 18. New York: Springer; 2014. p. 247-60.

20. InFACT: International Forum for Acute Care Specialists [Internet]. Available from: http://www.infactglobal.org/Home.aspx. [Assessed 2015 May 19]. 